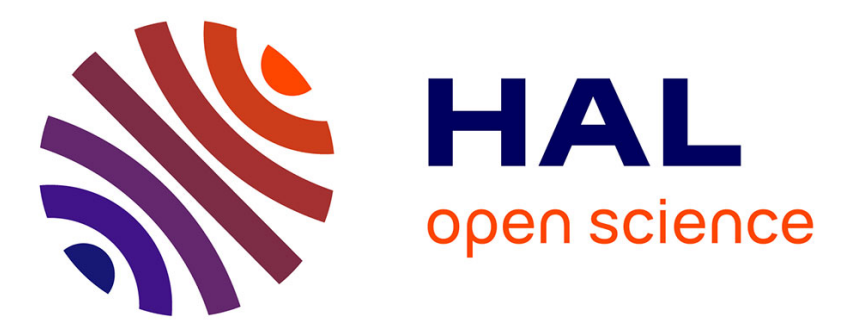

\title{
Optimize Wireless Networks for Energy Saving by Distributed Computation of Čech Complex
}

Ngoc-Khuyen Le, Anais Vergne, Philippe Martins, Laurent Decreusefond

\section{To cite this version:}

Ngoc-Khuyen Le, Anais Vergne, Philippe Martins, Laurent Decreusefond. Optimize Wireless Networks for Energy Saving by Distributed Computation of Čech Complex. The 13th IEEE International Conference on Wireless and Mobile Computing, Networking and Communications, WiMob 2017, 2017, Roma, Italy. hal-01580622

\section{HAL Id: hal-01580622 \\ https://hal.science/hal-01580622}

Submitted on 1 Sep 2017

HAL is a multi-disciplinary open access archive for the deposit and dissemination of scientific research documents, whether they are published or not. The documents may come from teaching and research institutions in France or abroad, or from public or private research centers.
L'archive ouverte pluridisciplinaire HAL, est destinée au dépôt et à la diffusion de documents scientifiques de niveau recherche, publiés ou non, émanant des établissements d'enseignement et de recherche français ou étrangers, des laboratoires publics ou privés. 


\title{
Optimize Wireless Networks for Energy Saving by Distributed Computation of Čech Complex
}

\author{
Ngoc-Khuyen Le, Anaïs Vergne, Philippe Martins, Laurent Decreusefond \\ LTCI, CNRS, Télécom ParisTech, Université Paris-Saclay, 75013, Paris, France \\ Email: \{ngoc-khuyen.le, anais.vergne, philippe.martins, laurent.decreusefond\}@telecom-paristech.fr
}

\begin{abstract}
In this paper, we introduce a distributed algorithm to compute the Cech complex. This algorithm is aimed at solving the coverage problems in self organized wireless networks. The complexity to compute the minimal Čech complex that gives information about coverage and connectivity of the network is $\mathcal{O}\left(n^{2}\right)$, where $n$ is the average number of neighbors of each cell. An application based on the distributed computation of the Čech complex, which is aimed at optimizing the wireless network for energy saving, is also proposed. This application also has polynomial complexity. The performance of the proposed algorithm and its application are evaluated. The simulation results show that the distributed computation of the Čech complex provides a consistent outcome with the one obtained by the centralized computation that is introduced in [6], while requires a much shorter calculation time. The optimized coverage saves $65 \%$ of the total transmission power, while also keeps the maximal coverage for the network.
\end{abstract}

\section{INTRODUCTION}

Coverage is a key factor that determines the quality of service in wireless networks. In recent applications to solve coverage problems in wireless networks, simplicial complex is often utilized to represent the network topology. Unlike graph, which represents only the neighborhood of cells, simplicial complex represents the topology of cells with a higher dimension.

The Cech complex is a simplicial complex that represents a collection of cells by a simplex if they have a non-empty intersection. The Čech complex represents exactly the topology of the network [3]. For this reason, the Čech complex is the right tool to describe and optimize the coverage for wireless networks. In [2], an algorithm to compute the Čech complex is introduced, but this algorithm is designed to be utilized in graphics science, and it only works with a collection of cells that have the same size. Nowadays, the wireless networks are becoming more and more dense and heterogeneous. This algorithm is not suitable to represent these networks. Although an algorithm to compute the Čech complex for a collection of cells that have different sizes is proposed in [6], this algorithm is still centralized. It is not suitable to represent future wireless networks, which should be self-organized.

In [7], authors use the Čech complex to optimize the coverage for wireless networks. As the computation of the Čech complex is only available in centralized way, this application is not distributed. In [5], [9], some algorithms based on simplicial homology are developed to detect coverage holes in wireless networks. Although these algorithms are available in both centralized and distributed ways, they use the Rips complex instead of the Čech complex to represent the topology of the network. However, the Rips complex is still an approximation of the Čech complex. In fact, the Rips complex represents a collection of cells by a simplex if every pair of cells in this collection are intersected (or they are neighbors). Therefore, the Rips complex may not capture exactly the topology of the network. So, some coverage holes may be undiscovered, see Figure 1 for an example. In this figure, a coverage hole inside three cells is represented by an empty triangle (not a 2 -simplex) in the Čech representation. It indicates that there is a hole inside three edges of the triangle. However, any pair of cells are intersected so the Rips complex represents these cells by a filled triangle (a 2-simplex). It means that no coverage hole is presented in the Rips representation. The Čech complex represents exactly the coverage hole, but the Rips complex does not.

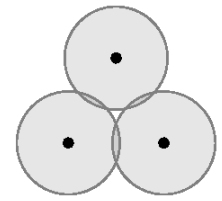

(a)

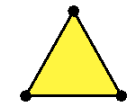

(b)

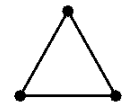

(c)
Fig. 1: (a) Cells, (b) Rips complex, (c) Čech complex.

In this paper, we introduce a distributed algorithm to compute the Čech complex for a given collection of cells that have different sizes. This algorithm is developed for solving coverage problems in self-organized wireless networks. The computation of the minimal Čech complex, which gives information about the coverage and connectivity of the network, is only $\mathcal{O}\left(n^{2}\right)$, where $n$ is the average number of neighbors of each cell. Following the simulation results, our distributed computation of the Cech complex provides a consistent outcome with the one obtained by the centralized computation that is introduced in [6], while requires much shorter computation time.

An example of the network deployment and its representation by the Cech complex is shown in Figure 2. The network in Figure 2a contains cells whose sizes are various. The center of each cell is marked as a black point. The covering domain of each cell is drawn in gray. In Figure 2b, every two neighbor cells are connected by a black edge (1-simplex). Each 2simplex is represented by a yellow triangle. Each 3 -simplex is drawn as a green tetrahedron, which represents the common 
intersection of 4 cells. The 4-simplices, which have the highest dimension, are drawn in red. The red areas present the most overlapped regions. The region that is not colored indicates a coverage hole.

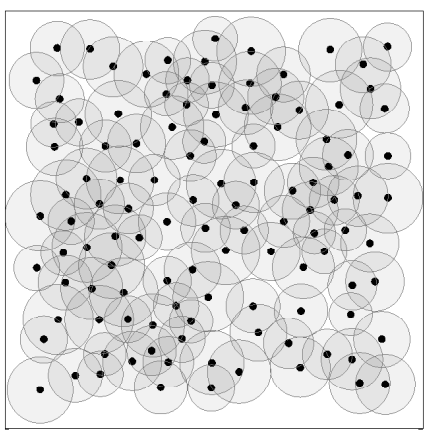

(a) A random deployment of cells

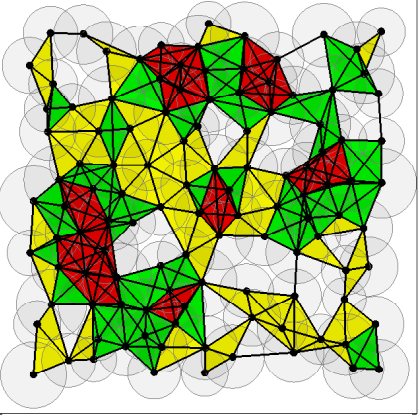

(b) Čech complex
Fig. 2: An example of the deployment of cells and its Čech complex

We also propose an application that is based on the distributed computation of the Čech complex. Given a random deployment of a wireless network, this application optimizes the coverage of the network. The optimized coverage reduces as much as possible the intersections among cells. As a result, the waste power due to interference within intersected regions is avoided efficiently. The total transmission power is reduced while the maximal coverage of the network is conserved. Our simulation results show that the optimized coverage saves $65 \%$ of the total transmission power in dense network. This application is also distributed and has only polynomial complexity.

This paper is organized as follows. Section II introduces a short background of simplicial homology and its applications in wireless networks. Section III describes all details about the distributed computation of the Čech complex. Section IV proposes a distributed coverage optimization algorithm aimed at energy saving for wireless networks. Section V shows our simulations and results. The last section concludes our paper with discussions and directions for future works.

\section{Simplicial HOMOLOGY}

This section introduces some notions of algebraic topology, and also explains how they can be used to capture and analyze the topology of the network. For more details about algebraic topology, see [8].

\section{A. Simplicial complex}

Let $S_{k}=\left\{v_{0}, v_{1}, \ldots, v_{k}\right\}$ be a set of $k+1$ geometrically independent points in $\mathbb{R}^{n}$, where $n>k$. The convex hull of $S$ is defined as a $k$-simplex, denoted by $s_{k}$, where $k$ is its dimension and $v_{0}, v_{1}, \ldots, v_{k}$ are its vertices. Thus, a 0 -simplex is a single point, an 1 -simplex is an edge, a 2 -simplex is a triangle, a 3-simplex is a tetrahedron, and so on. See Figure 3 for some instances.

The convex hull of any subset of $S_{k}$, which is also geometrically independent, is a lower dimensional simplex. This

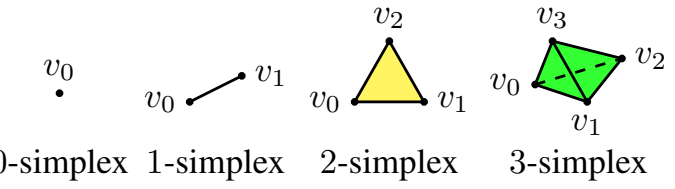

Fig. 3: An example of simplices.

simplex is called a $l$-face of $s_{k}$ if $l$ is its dimension. An abstract simplicial complex is a collection of simplices such that: every face of any simplex in this collection is also in this collection.

\section{B. Homology group}

We define the orientation of a simplex as an order of its vertices. A simplex $\left\{v_{0}, v_{1}, \ldots, v_{k}\right\}$ considering the order of its vertices is called an oriented simplex, denoted by $\left[v_{o}, v_{1}, \ldots, v_{k}\right]$. If the vertices of a simplex is transformed by an odd permutation, its orientation changes into the inverse one. If two vertices of a simplex swap their positions, then the orientation of this simplex is reversed, and denoted by the negative sign as:

$$
\left[v_{0}, \ldots, v_{i}, \ldots, v_{j}, \ldots, v_{k}\right]=-\left[v_{0}, \ldots, v_{j}, \ldots, v_{i}, \ldots, v_{k}\right] .
$$

Given $K$ an abstract simplicial complex, the $k$-chain group $C_{k}(K)$ is the vector space spanned by the set of oriented $k$ simplices of $K$. The boundary operator $\partial_{k}$ is defined as the linear transformation $\partial_{k}: C_{k}(K) \rightarrow C_{k-1}(K)$ which acts on basis elements $\left[v_{0}, v_{1}, \ldots, v_{k}\right]$ via:

$\partial_{k}\left[v_{0}, v_{1}, \ldots, v_{k}\right]=\sum_{i=0}^{k}(-1)^{i}\left[v_{0}, v_{1}, \ldots, v_{i-1}, v_{i+1}, \ldots, v_{k}\right]$,

where $\left[v_{0}, v_{1}, \ldots, v_{i-1}, v_{i+1}, \ldots, v_{k}\right]$ is the $i$-th face obtained by deleting the $i$-th vertex from $\left[v_{0}, v_{1}, \ldots, v_{k}\right]$. The boundary of a $k$-simplex is its collection of $(k-1)$-faces. It is simple to verify that $\partial_{k} \circ \partial_{k+1}=0$. This result means that the boundary of every chain has no boundary. A $k$-chain is called a $k$-cycle if its boundary is zero. So, the group of $k$-cycles, denoted by $Z_{k}(K)$, is the kernel of $\partial_{k}: C_{k}(K) \rightarrow C_{k-1}(K)$. The group of $k$-boundaries, denoted by $B_{k}(K)$, is the image of $\partial_{k+1}$ : $C_{k+1}(K) \rightarrow C_{k}(K)$. Since $\partial_{k} \circ \partial_{k+1}=0$ for every $k$, each $k$ boundary is automatically a $k$-cycle. It implies that $B_{k}(K) \subset$ $Z_{k}(K)$ for all $\mathrm{k}$. We now define the $k$-th homology group of $K$ as the quotient vector space: $H_{k}(K)=Z_{k}(K) / B_{k}(K)$. The dimension of $H_{k}$ is called the $k$-th Betti number:

$$
\beta_{k}=\operatorname{dim} H_{k}=\operatorname{dim} Z_{k}-\operatorname{dim} B_{k} .
$$

The Betti number has an important meaning in solving coverage problems. Given a simplicial complex that presents the coverage of a network, its $k$-th Betti number $\beta_{k}$ counts the $k$-dimensional holes. For example, while the connected components are counted by $\beta_{0}$, the coverage holes are counted $\beta_{1}$. Therefore, if $\beta_{0}=1$, the network is connected. There is no coverage hole if $\beta_{1}=0$.

\section{Simplicial complex of cellular networks}

Definition 1 (Čech complex): Given a collection of cover sets $\mathcal{U}$, the $\check{C}$ ech complex of $\mathcal{U}$, denoted as $\check{C}(\mathcal{U})$, is the 
abstract simplicial complex whose $k$-simplices correspond to nonempty intersection of $k+1$ distinct elements of $\mathcal{U}$.

If each cover set in the collection $\mathcal{U}$ is the coverage of a cell in the wireless network, then the Čech complex of $\mathcal{U}$ captures exactly the topology of this network [3].

\section{Distributed COMPUTATION OF ČECH COMPLEX}

\section{A. System model}

We consider a wireless network composed of $N$ distinct cells. We assume that each cell uses isotropic propagation. The coverage of the $i$-th cell is modeled as:

$$
c_{i}\left(v_{i}, r_{i}\right)=\left\{z \in \mathbb{R}^{2}:\left\|z-v_{i}\right\| \leq r_{i}\right\},
$$

where $\|$.$\| is the Euclidean distance, the vertex v_{i}$ represents the base station location and $r_{i}$ is the coverage radius of the $i$-th cell. Let $\mathcal{U}$ be the collection of cells, then $\mathcal{U}=\left\{c_{i}\right.$, for $i=$ $0,1, \ldots,(N-1)\}$. The Čech complex of $\mathcal{U}, \check{\mathbf{C}}(\mathcal{U})$, is defined as the Cech complex of the wireless network. In the Čech complex, each vertex, i.e. a 0 -simplex, $v_{i}$ corresponds to the $i$-th cell $c_{i}$ in the network. An edge, i.e. a 1-simplex, represents the connection, or the intersection, between two cells. Each $k$-simplex, where $k \geq 2$, represents the common intersection of the coverage of together $(k+1)$ corresponding cells of this simplex. For example, in Figure 4, the 2-simplex $\left[v_{2}, v_{3}, v_{6}\right]$ means the overlap of coverage of cell $c_{2}$, cell $c_{3}$ and cell $c_{6}$. There is no coverage hole inside these cells. The higher dimension of the simplex is, the higher number of overlaps is. The 3 -simplex $\left[v_{0}, v_{1}, v_{2}, v_{6}\right]$ means that the four corresponding cells: $c_{0}, c_{1}, c_{2}$ and $c_{6}$, together, have a common intersection. In contrast, a chain of 1-simplices indicates a coverage hole inside corresponding cells of the chain. For example, the chain $\left[v_{3}, v_{4}\right]+\left[v_{4}, v_{5}\right]+\left[v_{5}, v_{6}\right]+\left[v_{6}, v_{3}\right]$ covers a coverage hole inside four cells $c_{3}, c_{4}, c_{5}$ and $c_{6}$. To analyze the network topology, we use characteristics of the homology of the Čech complex.
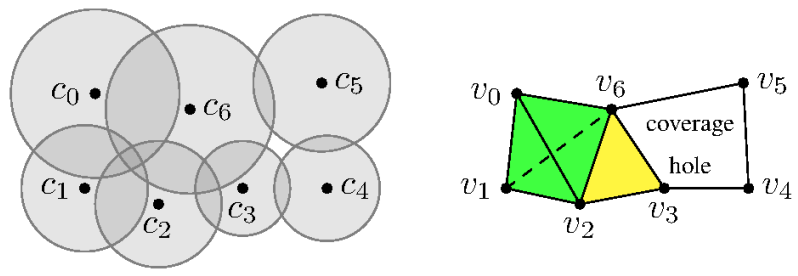

Fig. 4: Cells and their Čech representation.

\section{B. Distributed computation of the Čech complex}

In this subsection, we describe all the details about how each cell can cooperate with others to construct the Čech complex. We denote $\mathbf{S}_{k}$ the collection of all $k$-simplices of the complex. As the definition of the Čech complex, each $k$-simplex represents the common intersection of together its $(k+1)$ corresponding cells. Each vertex, that is a 0 -simplex, corresponds to a cell of the network. The collection of 0 simplices, $\mathbf{S}_{0}$, is then obviously the list of corresponding vertices of cells.

$$
\mathbf{S}_{\mathbf{0}}=\left\{v_{i} \mid i=0,1, \ldots,(N-1)\right\} .
$$

If a group of cells form a $k$-simplex, where $k \geq 1$, then every pair of cells in this group are neighbors. So, each cell needs to detect all its neighbors before computing the simplices.

1) Neighbors detection: We assume that each cell $c_{i}$ can communicate with other cells over radio within a distance $D_{i}=2 r_{i}$. We assume that there are enough frequency slots for cells to communicate over radio without collision. Every cell is also connected by a backhaul network. At the initial state, each cell broadcasts a probe message with its position and its radius over radio channel. If a cell receives a probe message, it verifies if the cell that sent this probe message is a neighbor. Two cells are neighbors if they are intersected. If they are neighbors, then the cell that received the probe message sends a relationship confirmation together with its position and its radius to the cell that sent the probe message by using the backhaul network. After receiving the confirmation, the cell that sent the probe message adds the cell that sent the confirmation into its collection of neighbors.

If two cells $a$ and $b$ are neighbors, the distance between them is $d(a, b) \leq r_{a}+r_{b}$. Let us assume that $a$ is the bigger cell, so $r_{a} \geq r_{b}$, then $d(a, b) \leq 2 r_{a}$ which is the communication distance $D_{a}$ of the cell $a$. So, the smaller cell $b$ always receives the probe message from the bigger cell $a$. Therefore, if there are two cells that are neighbors, the neighborhood is always detected by the smaller cell. We assume that all the cells can reply the confirmation within a period $t_{\text {ack }}$. After this period $t_{\text {ack }}$, every cell detects its collection of neighbors. We denote the collection of neighbors of the cell $c_{i}$ as $\mathbf{N}_{i}$.

2) Distributed simplices computation: When the collection of neighbors is available, each cell computes its simplices by verifying the intersection among it and its neighbors. As each pair of neighbors form a 1-simplex, the collection of 1simplices of each cell $c_{i}$ is easily found:

$$
\mathbf{S}_{\mathbf{1}, \mathbf{i}}=\left\{\left[v_{i}, v_{j}\right] \mid c_{j} \in \mathbf{N}_{i}\right\} .
$$

To find all $k$-simplices, where $k \geq 2$, each cell verifies if a group of it and its $k$ neighbors has a common intersection. If these cells have a common intersection, then this group forms a $k$-simplex.

We now consider a group of $(k+1)$ cells and verify if they form a $k$-simplex. Let us denote $\hat{c}_{l}\left(\hat{v}_{l}, \hat{r}_{l}\right)$ the considered cell in this group, where $l=0,1, \ldots, k$. The collection of vertices of these cells $\hat{s}_{k}=\left[\hat{v}_{0}, \hat{v}_{1}, \ldots, \hat{v}_{k}\right]$ is considered as a candidate to be a $k$-simplex. To decide if this $\hat{s}_{k}$ is a $k$-simplex, we now need to verify if all these corresponding cells $\hat{c}_{l}$, where $l=0,1, \ldots, k$, have a common intersection.

Let us assume that $\hat{s}_{k}$ is a $k$-simplex. It means that there is a common intersection of all these corresponding cells. We denote $\mathbf{I}$ to be this intersection, we have:

$$
\mathbf{I}=\cap \hat{c}_{l}, \text { for } l=0,1, \ldots, k .
$$

Let $p$ be a point that belongs to $\mathbf{I}$, then $p$ must belong to all corresponding cells $\hat{c}_{l}$, where $l=0,1, \ldots, k$. We denote circle $\hat{b}_{l}$ the boundary, or the cover, of the cell $\hat{c}_{l}$, and $\mathbf{X}$ the collection of intersection points of every pair of these circles.

$$
\mathbf{X}=\left\{\hat{b}_{m} \cap \hat{b}_{n} \mid 0 \leq m<n \leq k\right\} .
$$


There are only two possible cases:

- The first case: $\mathbf{X} \cap \mathbf{I}=\varnothing$. There is no intersection point that belongs to $\mathbf{I}$. In this case, the smallest circle, $\hat{b}_{\min }=$ $\min \left\{\hat{b}_{l} \mid l=0,1, \ldots, k\right\}$, must be inside all other circles $\hat{b}_{l}$ that $\hat{b}_{l} \neq \hat{b}_{\text {min }}$. See an example in Figure 5.
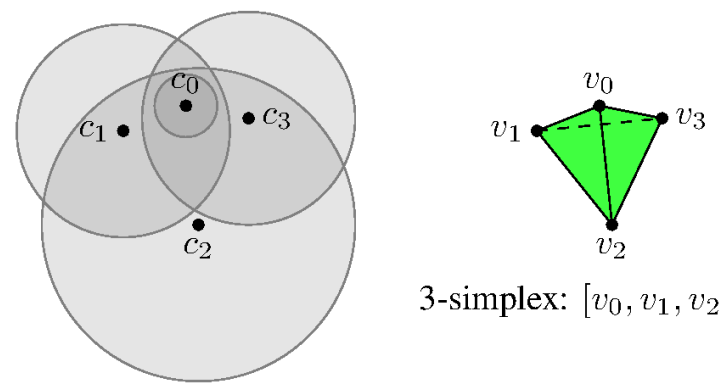

3-simplex: $\left[v_{0}, v_{1}, v_{2}, v_{3}\right]$

Fig. 5: The smallest cell is inside the other cells.

- The second case: $\mathbf{X} \cap \mathbf{I} \neq \varnothing$. There must exist two circles $\hat{b}_{m}$ and $\hat{b}_{n}$ (the boundary of $\hat{c}_{m}$ and $\hat{c}_{n}$ ), that at least one of their intersection points belongs to all other corresponding cells $\hat{c}_{l}$, where $l \neq m$ and $l \neq n$. See an example in Figure 6.
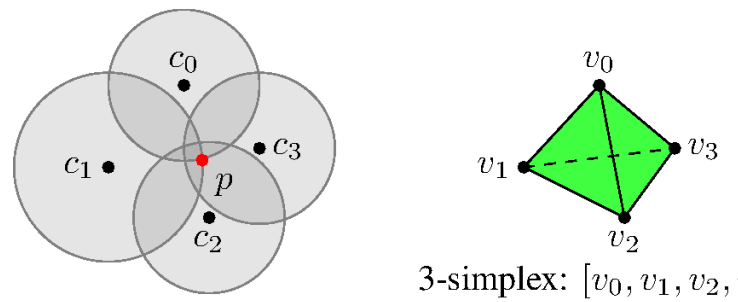

3-simplex: $\left[v_{0}, v_{1}, v_{2}, v_{3}\right]$

Fig. 6: There is a pair of cells whose one intersection point (the red point) is inside the other cells.

So, to verify a candidate to be a $k$-simplex, we need to verify if it satisfies one of these two cases above. If this candidate does not satisfy both of these two cases above, then it is not a $k$-simplex. See an example in Figure 7.
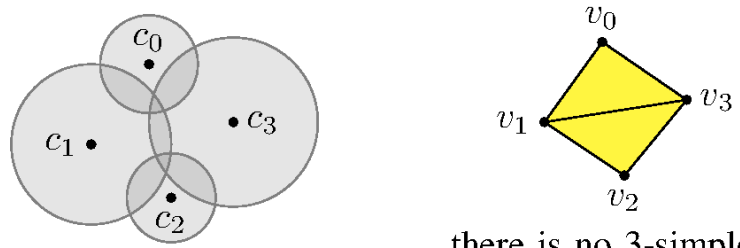

there is no 3-simplex

Fig. 7: The smallest cell is not inside the other cells, and no intersection point of any pair of two cells is inside the other cells.

However, neighborhood is a two-way relationship. Therefore, the verification of intersection could be duplicated by different cells that are neighbors. To avoid the redundant duplication, each cell verifies the intersection by following a right hand rule. This rule is that each cell verifies only with neighbors that are on its right hand side. If there is a neighbor which has the same horizontal coordinate, it verifies only this neighbor if it has higher vertical coordinate. If a simplex is found by a cell, this cell transmits this simplex to every cell that belongs to this simplex. As a result, every cell detects all its simplices. For example, in Figure 4 , the cell $c_{2}$ verifies the intersection with only the cell $c_{3}$ and $c_{6}$. It detects the simplex $\left[v_{2}, v_{3}, v_{6}\right]$. It receives its other simplices from the neighbor $c_{0}$ and $c_{1}$. The Algorithm 1 reports the distributed computation of Čech complex for each cell. In this algorithm, we denote $c_{i}$ the cell that is computing the simplices and $\left(x_{i}, y_{i}\right)$ its coordinates. The parameter "count_time" counts time up from

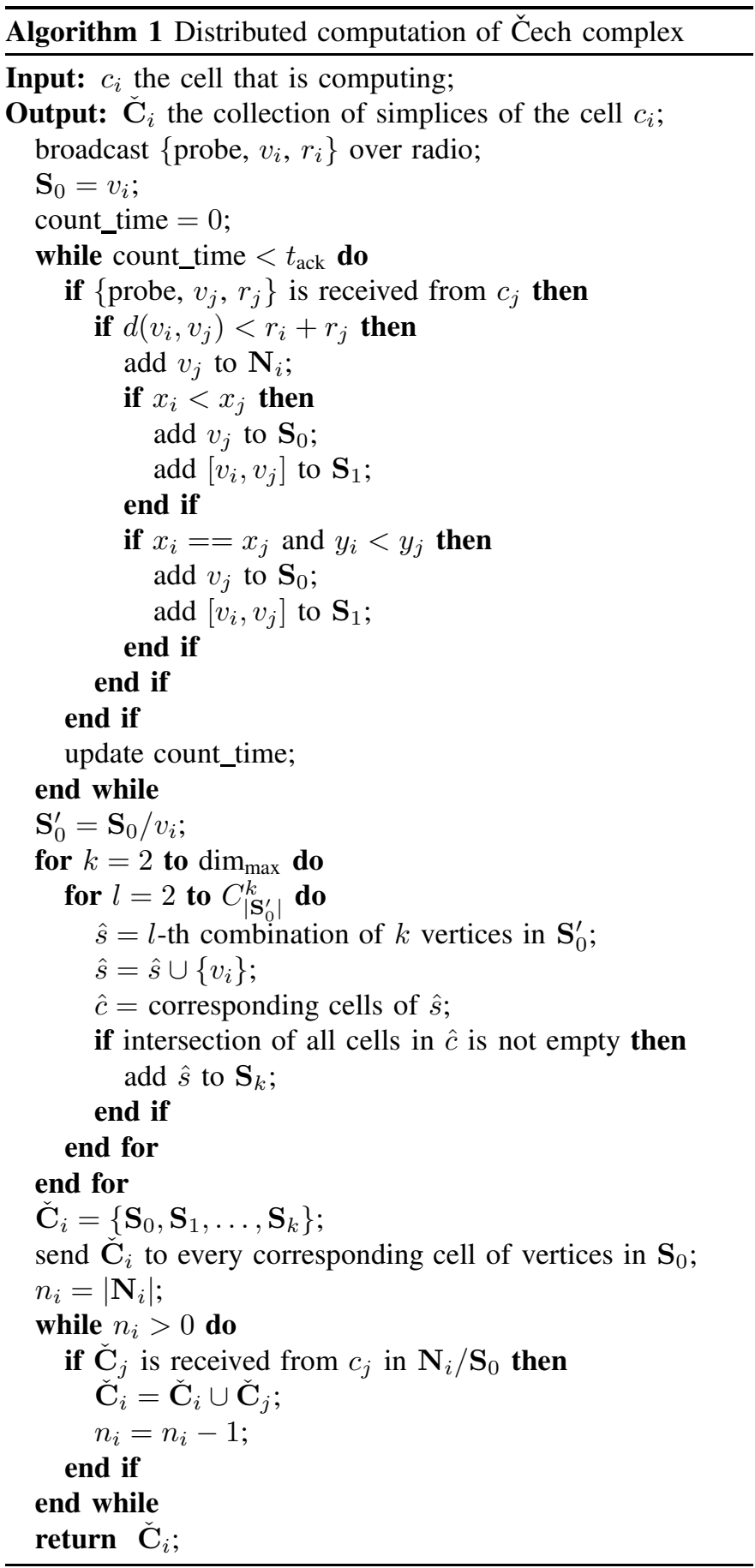

the moment the cell $c_{i}$ broadcasts the probe message. The highest dimension of simplices that are considered is $\operatorname{dim}_{\max }$. The output of this algorithm, the collection of simplices of the cell $c_{i}$ is denoted as $\check{\mathbf{C}}_{i}$. 
The global Čech complex that represents the topology of the whole network is sometimes needed. There should be a master cell that controls the topology of the network. This global Čech complex $\check{\mathbf{C}}(\mathcal{U})$ can be easily built by integrating the simplices computed from every cell as:

$$
\check{\mathbf{C}}(\mathcal{U})=\cup_{i=0}^{N-1} \check{\mathbf{C}}_{i} .
$$

Each cell sends its computed simplices that contain only the vertices satisfied the right hand rule. One more time, this rule is useful as it avoids sending the duplicated simplices.

\section{Complexity}

We denote the average number of neighbors of each cell by $n$. To find a $k$-simplex, each cell needs to verify the common intersection of a group of it and its $k$ neighbors. To find all $k$-simplices, the number of groups to verify is $C_{n}^{k}$. So, to find all $k$-simplices for $1 \leq k \leq d_{\max }$, the number of groups to verify is $\sum_{k=1}^{d_{\max }} C_{n}^{k}$.

If we consider only the connectivity and coverage of the wireless network, the Čech complex built up to dimension two is enough. In this case, the complexity to find all simplices up to dimension two of each cell is $\mathcal{O}\left(n^{2}\right)$. If we consider the Čech complex with the highest dimension $d_{\max }$, assuming that $d_{\max }=\infty$, the sum $\sum_{k=1}^{d_{\max }} C_{n}^{k}$ is upper bounded by $2^{n}$. So, the complexity to find all the simplices up to the highest dimension is $\mathcal{O}\left(2^{n}\right)$. Although it is not polynomial, the term $2^{n}$ is not tremendous because the average number of neighbors of each cell is normally not so big. The complexity of our distributed construction of the Čech complex is much smaller than the one of the centralized construction proposed in [6]. The complexity to build the Čech complex up to dimension two and up to its highest dimension by the centralized construction are $\mathcal{O}\left(N^{2}+\right.$ $\left.N n^{2}\right)$ and $\mathcal{O}\left(N^{2}+N 2^{n}\right)$, respectively.

\section{COVERAge OPtIMIZATION FOR ENERGy SAVING}

One application of the distributed computation of the Čech complex is coverage optimization for energy saving in wireless networks. Our proposal jointly maximizes its coverage and minimizes its total transmission power. Firstly, we ensure a maximal coverage for the network. Each cell is turned on and is set to work with the highest transmission power. At this initial state, the network has the largest coverage. However, many cells are hardly overlapped. The overlapping region between cells causes the waste of transmission power due to interference. We can optimize the transmission power by reducing the overlapping regions. However, the global coverage of the network should be conserved. In other words, the two Betti numbers $\beta_{0}$ and $\beta_{1}$ of the Čech complex of the network should be kept unmodified. The transmission power of each cell is estimated following the simplified path loss model:

$$
P_{t, i}=K_{0} r_{i}^{\gamma}
$$

where $K_{0}$ is a constant factor and $\gamma$ is the path loss exponent. In this paper, $K_{0}$ is assumed to be 1 for simplification. The optimization problem can be written as:

$$
\begin{array}{ll}
\min _{r} & \sum_{i=0}^{N-1} r_{i}^{\gamma} \\
\text { s.t. } & \beta_{0}=\beta_{0}^{*} \\
& \beta_{1}=\beta_{1}^{*} \\
& r=\left(r_{0}, r_{1}, \ldots, r_{N-1}\right),
\end{array}
$$

where $\beta_{0}^{*}$ and $\beta_{1}^{*}$ are the Betti numbers of the Čech complex of the network at the initial state.

We introduce a distributed algorithm to optimize the coverage as well as to save energy for the network. This algorithm is applied for each cell in the network. We assume that all the fenced cells and boundary cells are already known. Only the cells that are not fenced or boundary cells can try to reduce the coverage radius. We assume that each cell $c_{i}$ in the network can be active with a coverage radius $r_{i}$, where $r_{i, \min } \leq r_{i} \leq r_{i, \min }$. If after the optimization process $r_{i} \leq r_{i, \min }$, the cell $c_{i}$ is turned off. We also assume that every cell is connected to each other by a backhaul network.

At the first step, each cell needs to search for its neighbors as well as its simplices by following the Algorithm 1. Once the neighbors set is established, each cell starts its reduction process. On each cell, there is a timer which counts down to zero. The timer is set to a uniform random value from 1 to $t_{\max }$, where $t_{\max }$ is the maximal value of the timer. When the timer of a cell is expired, this cell tries to do a reduction. If two cells that are neighbors try to reduce their radius at the same time, the coverage hole may not be detected due to the outdated information about the radius of each other. Therefore, before trying to reduce the radius, each cell sends a "pause" message to its neighbors. Then, the cell reduces its coverage radius and verifies the coverage.

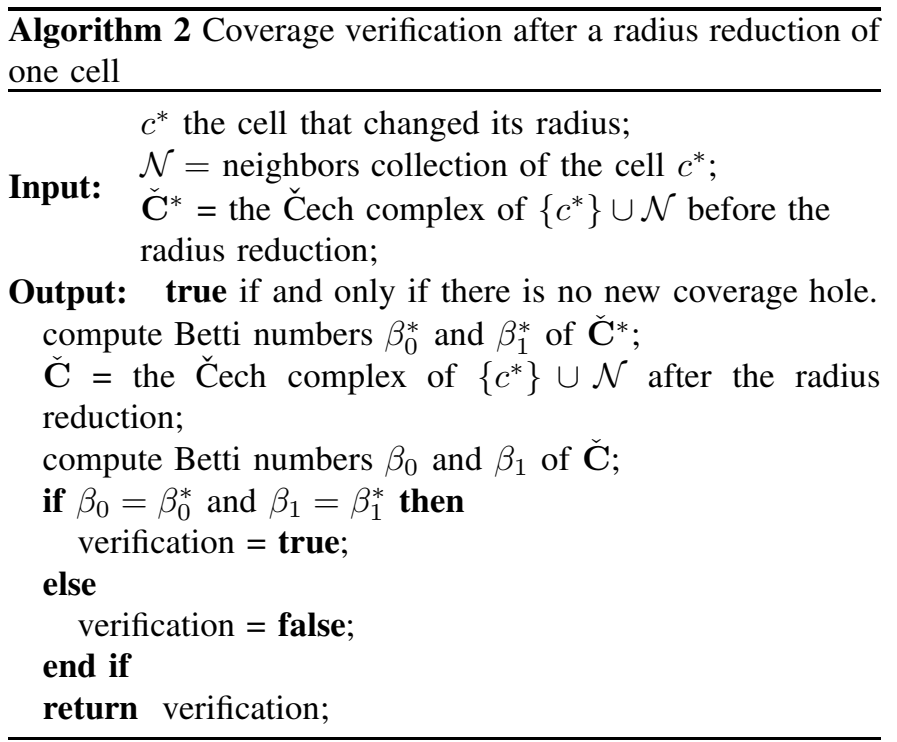

The radius reduction of one cell only makes topology change in the local region that is comprised of this cell and 
its neighbors. If there is a new coverage hole, it must be inside this local region. This means that if there is no new coverage hole after the radius reduction, the Betti numbers $\beta_{0}$ and $\beta_{1}$ of the Čech complex of this local region are unchanged. The verification of the network coverage can be reduced to the coverage verification in only this local region as in the Algorithm 2.

If no hole appears, the cell confirms the reduction and sends the new value of coverage radius to its neighbors. It also sends the "continue" message to its neighbors to tell them that they can continue. If a cell received a "pause" message, it pauses its process and waits until the message "continue" is received. Then, it continues its process normally.

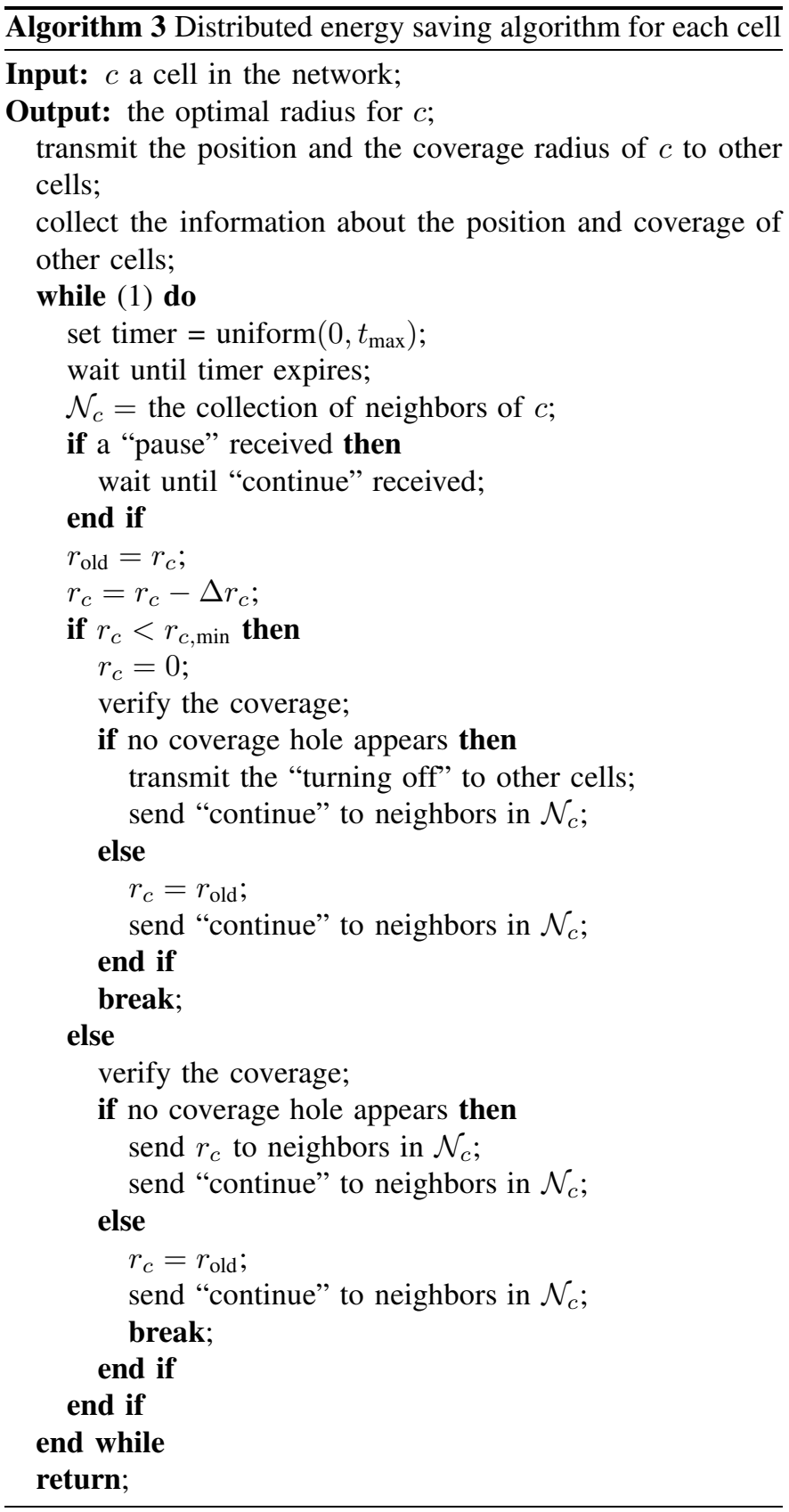

There is a special case where two neighbor cells whose timers expire at the same time send the "pause" message to each other simultaneously. One of these two cells receives a "pause" from another before it sends "continue" message to other cells. This cell cancels the current reduction step and sets a new value for its timer and waits to retry.

If a cell tries to reduce its coverage radius and makes a coverage hole, it reverses its coverage radius to the previous value and stops the reduction process. This cell is set to irreducible.

The distributed energy saving algorithm applied for each cell is described in the Algorithm 3. The main step in this algorithm is to verify the coverage of the local region. This step requires the computation of the Cech up to dimension 2 and its Betti numbers $\beta_{0}, \beta_{1}$. The computation of this Čech complex has complexity $\mathcal{O}\left(n^{2}\right)$. The computation of the two Betti numbers $\beta_{0}$ and $\beta_{1}$ are based on the rank computation of the homology space of the Čech complex. The complexity of these computations are discussed in [4]. Let we denote $m_{k}$ the average number of $k$-simplices of the Čech complex. The computation of $\beta_{0}$ has the complexity of $\mathcal{O}\left(m_{1}\right)$. The complexity of the computation of $\beta_{1}$ is equal to the complexity of the rank computation of a square matrix $m_{2}$ rows and $m_{2}$ columns, which is $\mathcal{O}\left(m_{2}^{3}\right)$. The number of $k$-simplices in the Čech complex can be upper bounded by $C_{n+1}^{k+1}$. So, the complexity to compute both these two Betti numbers $\beta_{0}$ and $\beta_{1}$ is then as much $\mathcal{O}\left(n^{9}\right)$. It is much smaller than the complexities of the centralized energy saving algorithm and the simulated annealing algorithm, which are $\mathcal{O}\left(N^{4} n^{6}\right)$ and $\mathcal{O}\left(K L N^{3} n^{6}\right)$, respectively [7]. In these terms, $N$ is much bigger than $n$. The number $K$ and $L$ are parameters of temperature schedule, which are set to a great value for a good approximation of the global optimum solution [1].

\section{Simulation AND RESUlts}

\section{A. Performance of the distributed computation of the Čech complex}

We compare the performance of our distributed construction of the Čech complex with the centralized one which is proposed in [6]. Cells are randomly deployed according to the Poisson point process on a square $[30 \times 5]$. The density of cells is varied from 1 (medium) to 2 (high). The radius of each cell is a random variable from 0.5 to 1 . The Čech complex is built up to dimension 2 (the minimal one that gives information about coverage and connectivity), and up to its highest dimension $d_{\max }$. The simulation is repeated 1000 times. The Table I notes the average simplices computation time (the period of neighbors detection $t_{\text {ack }}$ is not included).

TABLE I: Construction time (second) of the Čech complex.

\begin{tabular}{crrrrr}
\hline \multirow{2}{*}{ Density of cells } & \multicolumn{2}{c}{ Centralized computation } & & \multicolumn{2}{c}{ Distributed computation } \\
\cline { 2 - 3 } \cline { 5 - 6 } & $d=2$ & $d=d_{\max }$ & & $d=2$ & $d=d_{\max }$ \\
\hline 1.0 & 0.144 & 0.526 & & 0.005 & 0.053 \\
1.5 & 0.946 & 11.825 & & 0.006 & 1.433 \\
2.0 & 4.088 & 218.290 & & 0.007 & 26.479 \\
\hline
\end{tabular}

As we can observe, the construction time increases quickly when we increase the density of cells. Because, both the 
number of cells $N$ and the average number of neighbors of each cell $n$ increase with the density of cells. In addition, the complexity to construct the Cech complex up to its highest dimension increases exponentially with $n$ (see the subsection III-C). The distributed algorithm saves much computation time, due to their lower complexity of computation.

The number of transmissions that are sent by each cell, as well as the size of each transmission are important to evaluate the performance of the distributed construction of the Cech complex. The message probe is sent only once by each cell. If a cell receives a probe message and it detects that the sender is a neighbor, then it sends an acknowledgment message. This message has a constant size, it contains only its id, its position $(x, y)$, and its radius of coverage. We assume that each of these values is represented by 4 bytes, the size of this message is 16 bytes, which is small. So, we consider only the number of acknowledge messages (ACKs) that are sent by each cell. After the construction of the local complex, each cell sends its list of simplices following the right hand rules. This list may be long and its size is not constant. We write this list to a text file, where each cell's vertex in a simplex is separated by a space and each simplex in the list is separated by a comma. The size of each character in this text is one byte. In the Table II, we note the average number of ACKs that are sent by each cell, as well as the average number of transmissions needed for each cell to exchange the simplices, and the average size (in bytes) of each transmission.

TABLE II: Performance of the distrbuted construction of the Čech complex.

\begin{tabular}{|c|c|c|c|c|c|}
\hline \multirow{2}{*}{$\begin{array}{l}\text { Density } \\
\text { of cells }\end{array}$} & \multirow{2}{*}{$\begin{array}{l}\text { Number } \\
\text { of ACKs }\end{array}$} & \multicolumn{2}{|c|}{ Number of transmissions } & \multicolumn{2}{|c|}{ Size of a transmission (bytes) } \\
\hline & & $d=2$ & $d=d_{\max }$ & $d=2$ & $d=d_{\max }$ \\
\hline 1.0 & 5.52 & 3.05 & 3.03 & 23.59 & 20.14 \\
\hline 1.5 & 8.32 & 4.59 & 4.55 & 38.82 & 48.61 \\
\hline 2.0 & 11.01 & 6.07 & 6.10 & 45.43 & 170.01 \\
\hline
\end{tabular}

At the highest density of cells, each cell needs only about 6 transmissions to exchange the list of simplices. If the Čech complex is built up to dimension 2, the size of each transmission is increased slowly with the density of cells. If the Cech complex is built up to its highest dimension, the size of each transmission is increased faster.

\section{B. Performance of the distributed energy saving algorithm}

We introduce firstly an example of wireless network before and after the coverage optimization in Figure 8. In Figure 8a, the cells of the network are not optimized. Many cells overlap and the transmission power is wasted. In Figure 8b, the cells of the network are optimized. Some cells are turn off, the remaining cells work with optimized coverage radius. The overlapping region is minimized. As a result, it prevents the wasted transmission power. The transmission power of the network is reduced but the global coverage is kept unchanged.

The performance of our distributed energy saving is evaluated. It is also compared with the one obtained by the centralized energy saving algorithm and the simulated annealing algorithm that are proposed in [7]. The simulated annealing

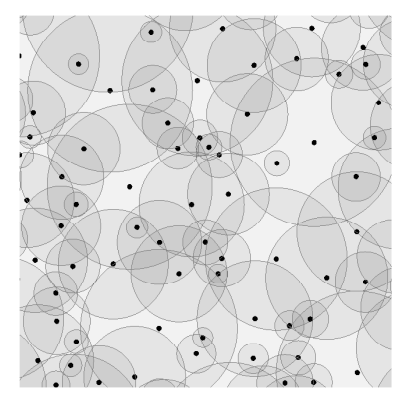

(a) Cells before the optimization

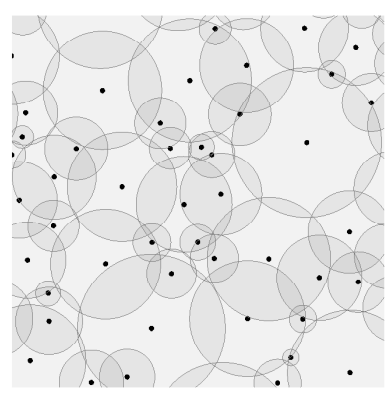

(b) Cells after the optimization
Fig. 8: Cells before and after the optimization

algorithm finds the global optimized solution. However, it has very big complexity and is not used in practice. The centralized one finds only a local minimum solution but it has smaller complexity.

We deploy the cells on a space $[10 \times 10]$ according to the Poisson point process. The density of cells is set to different values from 0.2 to 1 . The coverage radius of each cell can vary from $r_{\min }=0.1$ to $r_{\max }=1$. We assume that the path loss exponent $\gamma$ is 3 . The Čech complex with dimension two is enough to get the information about coverage and connectivity of the wireless network. So we do not need to build it up to a higher dimension as it causes longer computation time for the same information. Our simulations are repeated 1000 times.

1) Consumed power of optimized cells: Before the optimization, each cell is set to work with its maximum coverage radius $r_{\max }=1$. At this state, each cell transmits the maximal power, which is 1, following the Equation 2. After performing the energy saving algorithms, the average consumed power per cell with the optimized radius is shown in Figure 9.

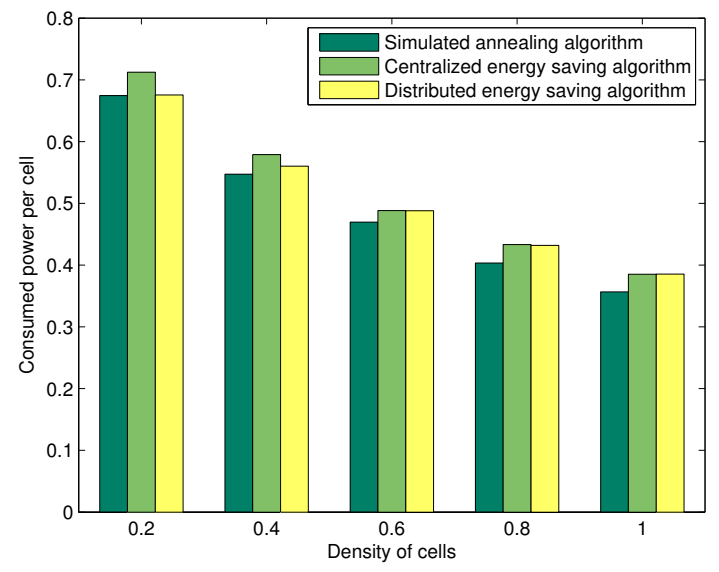

Fig. 9: Consumed power of optimized cells by different algorithms

The simulation shows that our distributed energy saving algorithm has better performance than the the centralized energy saving algorithm proposed in [7] at low density of cells. It also has the same performance with the centralized one at the high density of cells. The difference between the performance of our distribued energy saving algorithm with the one of the simulated annealing algorithm [7] is always less than 3\%. However, our distributed energy saving algorithm has 
much smaller complexity. At the highest density of cells, the cells optimized by the simulated annealing algorithm operate with $35 \%$ their original power, thus it saves $65 \%$ power. Our distributed algorithm saves $62 \%$ power.

2) Probability density function of optimized cells' radius: We consider the pdf of the optimized radius of cells at different densities: high (1.0), medium (0.6), and low (0.2). They are presented in Figure 10, Figure 11, and Figure 12, respectively. While the pdf of cells radius optimized by our distributed energy saving algorithm and the centralized one [7] are quite similar, they are different from the one obtained by the simulated annealing algorithm [7]. The distributed and centralized energy saving algorithms turn off many cells. They turn off $35 \%$ and $10 \%$ of cells when the cells densities are high and low, respectively. The simulated annealing algorithm turns off less than $10 \%$ of cells at every density of cells.

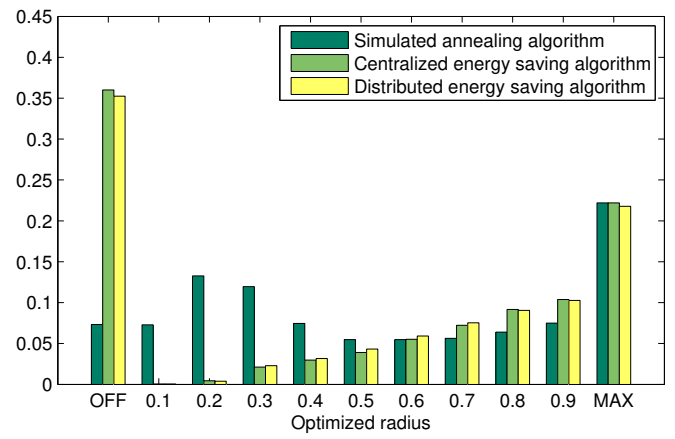

Fig. 10: Pdf of optimized radius at high density of cells (1.0)

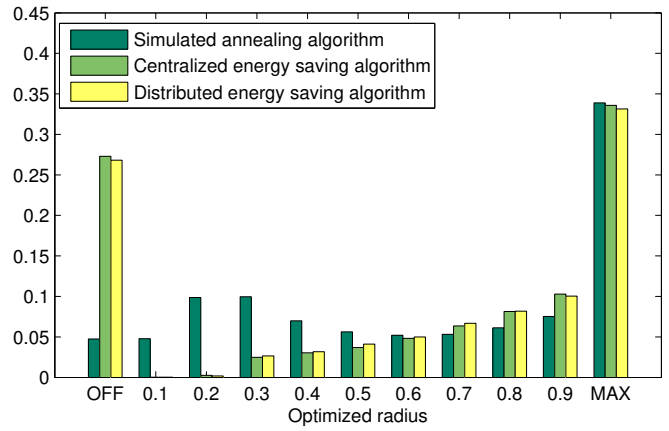

Fig. 11: Pdf of optimized radius at medium density of cells (0.6)

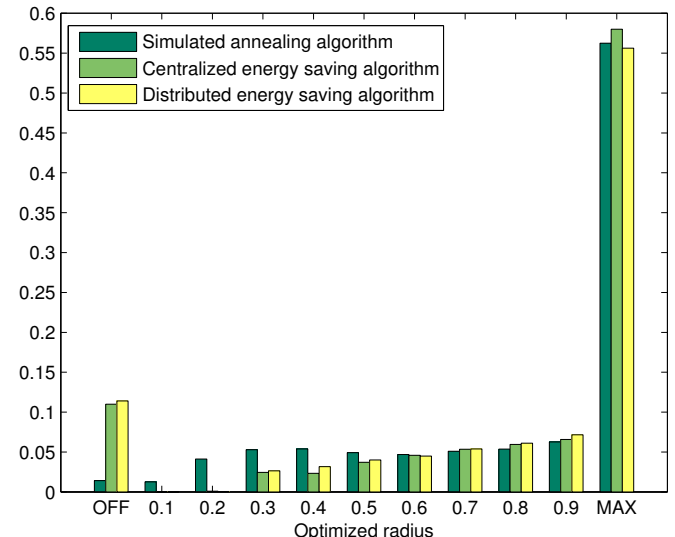

Fig. 12: Pdf of optimized radius at low density of cells $(0.2)$
The distributed and centralized algorithms avoid to keep active small cells while the simulated annealing one remains them. The advantage of the distributed energy saving algorithm is that it provides a similar performance as this simulated annealing one, while it requires a smaller number of active cells and has the smallest complexity.

The number of medium sized cells are quite similar at different densities of cells. There are $56 \%$ of cells work with the maximal coverage at low density of cells, but this number is only $22 \%$ at high density of cells. It means that the medium cells are substantial while the larger cells should be reduced in dense networks.

\section{CONCLUSION}

This paper introduces the distributed computation of the Čech complex aimed for self organized wireless networks. The complexity to compute the minimal Čech complex, which gives information about coverage and connectivity, is polynomial. A distributed application based on this distributed computation of the Čech complex is also proposed. This application aims at coverage optimization for energy saving in wireless networks. It has the same performance as the one of the centralized energy saving in [7] while having smaller complexity. The optimized network saves transmission power and only requires a small number of active cells, which allows to cut down the deployment costs.

Although homology theory gives information about coverage and connectivity, it still locates and counts holes without measuring them. Its extension called persistent homology theory allows multi-scale analysis of the coverage of wireless networks. Applications of the Čech complex can be more developed with persistent homology theory.

\section{REFERENCES}

[1] Dimitris Bertsimas and John Tsitsiklis. Simulated annealing. Statist. Sci., 8(1):10-15, 021993.

[2] Stefan Dantchev and Ioannis Ivrissimtzis. Efficient construction of the ech complex. Computers \& Graphics, 36(6):708 - 713, 2012. 2011 Joint Symposium on Computational Aesthetics (CAe), Non-Photorealistic Animation and Rendering (NPAR), and Sketch-Based Interfaces and Modeling (SBIM)

[3] Vin de Silva, Robert Ghrist, and Abubakr Muhammad. Blind swarms for coverage in 2-D. In Proceedings of Robotics: Science and Systems, Cambridge, USA, June 2005.

[4] H. Edelsbrunner and S. Parsa. On the computational complexity of betti numbers: Reductions from matrix rank. In Proceedings of the TwentyFifth Annual ACM-SIAM Symposium on Discrete Algorithms, SODA '14, pages 152-160. SIAM, 2014.

[5] Robert Ghrist and Abubakr Muhammad. Coverage and hole-detection in sensor networks via homology. In Proceedings of the 4th International Symposium on Information Processing in Sensor Networks, IPSN '05, Piscataway, NJ, USA, 2005. IEEE Press.

[6] Ngoc-Khuyen Le, P. Martins, L. Decreusefond, and A. Vergne. Construction of the generalized Cech complex. In Vehicular Technology Conference (VTC Spring), 2015 IEEE 81st, pages 1-5, May 2015.

[7] Ngoc-Khuyen Le, P. Martins, L. Decreusefond, and A. Vergne. Simplicial homology based energy saving algorithms for wireless networks. In Communication Workshop (ICCW), 2015 IEEE International Conference on, pages 166-172, June 2015.

[8] James Munkres. Elements of algebraic topology. Perseus Books, 1984.

[9] Feng Yan, P. Martins, and L. Decreusefond. Connectivity-based distributed coverage hole detection in wireless sensor networks. In Global Telecommunications Conference (GLOBECOM 2011), 2011 IEEE, pages 1-6, Dec 2011. 